\title{
Diacronie
}

Studi di Storia Contemporanea

$\mathrm{N}^{\circ} 19,3 \mid 2014$

Miscellaneo

\section{Nedret Kuran-Borçoğlu, Reflections on the Image of the Turk in Europe}

\section{Luca Zuccolo}

\section{Q OpenEdition \\ 1 Journals}

Edizione digitale

URL: http://journals.openedition.org/diacronie/1652

DOI: 10.4000/diacronie. 1652

ISSN: 2038-0925

Editore

Association culturelle Diacronie

Notizia bibliografica digitale

Luca Zuccolo, « Nedret Kuran-Borçoğlu, Reflections on the Image of the Turk in Europe », Diacronie

[Online], $N^{\circ} 19,3$ | 2014, documento 9, Messo online il 01 septembre 2014, consultato il 23 septembre 2020. URL : http://journals.openedition.org/diacronie/1652 ; DOI : https://doi.org/10.4000/diacronie. 1652

Creative Commons License 


\section{RECENSIONE:}

\section{Nedret KURAN-BORÇOĞLU, Reflections on the Image of the Turk in Europe, Istanbul, ISIS Press, 2009, 100 pp.}

a cura di Luca ZUCCOLO *

Negli ultimi anni la Turchia e i Turchi sono saliti sempre più spesso all'onore della cronaca politica nei principali organi di stampa europei. I fatti della Mavi Marmara (2010) e quelli di Gezi Park (2013) hanno riportato l'attenzione della Comunità Europea e dell'opinione pubblica continentale sulla Turchia e la sua città simbolo, Istanbul, riconducendole nuovamente al centro del dibattito europeista. Un dibattitto che a partire dalla decisione di riaprire i negoziati per l'adesione all'Unione europea (2005) ha vissuto, sino ad oggi, notevoli stravolgimenti e cambiamenti, dovuti sia alle azioni politiche interne ed estere del Governo di Ankara, sia ad una serie di pregiudizi europei non ancora superati e sempre pronti a riemergere.

Nel primo decennio del nuovo millennio, infatti, se da un lato la Turchia e il suo popolo si sono fatti conoscere e apprezzare da numerosi punti di vista, dall'altro lato la maggiore esposizione del Paese e dei suoi politici in ambito europeo ha fatto riaffiorare alcuni vecchi stereotipi che si ritenevano sopiti. L'immagine del Turco in Europa ha vissuto, fin dai primi contatti tra questi due "mondi", una serie pressoché infinita di contrasti e riappacificazioni in cui si riflettevano le paure e le aspettative degli stessi Europei. Un importante strumento per comprendere questa visione plurima e, a volte, distorta, dei Turchi in Europa è la ricerca imagologica e, in modo particolare, il saggio di Nedret Kuran-Burçoğlu qui presentato.

Docente della Boğaziçi University ed attualmente in carica alla Yeditepe University di Istanbul, Nedret Kuran-Burçoğlu - specializzata nel campo degli studi culturali comparati, della letteratura, dell'imagologia e degli studi transnazionali e 
transculturali ${ }^{1}$ - propone in questa raccolta di articoli il frutto di anni di studio sull'immagine dei Turchi e sulla sua ricezione in Europa tra il XV e il XX secolo.

Il volume, attraverso cinque agili articoli, si snoda attraverso la longue durée della storia Ottomano-turca evidenziando alcune fasi salienti della ricezione e dell'immaginario del turco in Europa, facendone emergere chiaramente la pluralità e la complessità. Il primo dato che emerge dalla lettura di questi articoli è la diversa visione dei Turchi maturata dagli Europei nel corso dell'Età Moderna. Una diversità di visioni e di opinioni motivata sia dalla distanza geografica, come nel caso dell'Inghilterra, sia dalle motivazioni politiche e religiose, come nel caso dello spazio linguistico tedesco (capitolo 1). Se le relazioni politiche e commerciali, così come gli scontri bellici e religiosi possono influire sull'approccio all'alterità rendendola di volta in volta negativa o positiva, l'immagine dei Turchi arriva in Europa anche tramite altri canali: in particolar modo attraverso la cultura. Il secondo articolo, infatti, si concentra sullo sviluppo della tipografia Ottomana descrivendo l'esperienza di Ibrahim Mütefferika nel XVIII secolo e la ricezione europea della vita intellettuale turca. Una ricezione che testimonia l'integrazione dell'Impero Ottomano nel sistema euro-mediterraneo e la partecipazione condivisa ad uno stesso panorama culturale.

L'evoluzione dell'immagine dei Turchi in Europa, passata attraverso rappresentazioni carnascialesche, opere teatrali, racconti dei viaggiatori e le invettive luterane, si arricchisce poi di un altro aspetto, quello visivo- Questo è il tema proposto nel terzo intervento, attraverso il confronto tra le memorie di Jean-Baptiste Vanmour e Jean Léon-Gérome, due pittori con un'opposta visione del Turco: qui realtà (JeanBaptiste Vanmour) e immaginazione (Jean Léon-Gérome) si intrecciano dimostrando la pluralità del mondo turco e delle sue interpretazioni in ambito europeo. Interpretazioni stereotipate e idealizzate, che sovente sono frutto dell'assenza di contatti diretti con i Turchi e con il loro mondo. Un'assenza sottolineata anche nel tema del quarto capitolo, in cui l'autrice analizza l'immagine dei Turchi nella novella Von Bagdad nach Stamboul dello scrittore tedesco, del XIX secolo, Karl May.

La letteratura turco-tedesca, è anche il tema dell'ultimo articolo, in cui KuranBurçoğlu analizza l'esperienza degli scrittori turchi emigrati in Germania e la loro diversa rappresentazione di se stessi e della cultura di ricezione lungo tre generazioni.

\footnotetext{
${ }^{1}$ Presidente del Crossroads Interdisciplinary Research and Policy Platform, fondato nel 2001 a Istanbul, Nedret Kuran-Burçoğlu è parte di cinque progetti internazionali sul multiculturalismo e tra le sue principali pubblicazioni si annoverano il libro History of Printing in the Ottoman Empire, traduzione del volume di Franz Babinger (2004); KURAN-BORÇOĞLU, Nedret (ed.), Multiculturalism: Identity and Otherness, Istanbul, Boğaziçi University, 1997; ID., The Image of the Turk in Europe from 1923 to 1990s, Istanbul, ISIS Press, 2000.
} 
Il saggio, infine, si chiude con un breve compendio dell'immagine del Turco in Europa e un'utile bibliografia selezionata delle pubblicazioni edite sull'argomento, che si unisce e completa i riferimenti bibliografici dei singoli articoli e l'ampio apparato fotografico.

Il volume, breve e di semplice comprensione, si propone come un ottimo punto di partenza per lo studio del tema in oggetto, ma anche un ottimo strumento per conoscere meglio la storia europea, quella ottomano-turca e, soprattutto, le loro relazioni. Nel complesso rapporto tra Europa e Turchia, infatti, la possibilità di una più approfondita comprensione dei rapporti culturali tra queste due realtà e la loro costante interconnessione risulta essere di fondamentale importanza per il superamento degli atavici stereotipi, oramai privi di fondamento e di senso, che troppo frequentemente riappaiono in molte immagini contemporanee del Turco in Europa. 


\section{* L'autore}

Luca Zuccolo è dottore in Storia Contemporanea presso il SUM (Istituto Italiano di Scienze Umane) di Napoli. Già dottore magistrale in Storia d'Europa (Bologna, 2008), il suo campo di ricerca si rivolge allo sviluppo della modernità durante l'ultimo secolo dell'Impero Ottomano, al confronto/scontro tra modernità e tradizione in un contesto cosmopolita e allo sviluppo dei movimenti sociali che hanno preparato l'avvento della società turca contemporanea. È il referente di Diacronie per la storia turca e ottomana.

URL: < http://www.studistorici.com/2010/12/o1/luca-zuccolo >

\section{Per citare questo articolo:}

ZUCCOLO, Luca, «Recensione: Nedret KURAN-BORÇOĞLU, Reflections on the Image of the Turk in Europe, Istanbul, ISIS Press, 2009, 100 pp.», Diacronie. Studi di Storia Contemporanea, 29/09/2014,

URL:< http://www.studistorici.com/2014/09/29/zuccolo_numero_19/ >

Diacronie Studi di Storia Contemporanea $\beta$ www.diacronie.it

Risorsa digitale indipendente a carattere storiografico. Uscita trimestrale. redazione.diacronie@hotmail.it

Comitato di redazione: Jacopo Bassi - Luca Bufarale - Elisa Grandi - Deborah Paci - Fausto Pietrancosta - Matteo Tomasoni - Luca Zuccolo

Diritti: gli articoli di Diacronie. Studi di Storia Contemporanea sono pubblicati sotto licenza Creative Commons 2.5. Possono essere riprodotti a patto di non modificarne i contenuti e di non usarli per fini commerciali. La citazione di estratti è comunque sempre autorizzata, nei limiti previsti dalla legge. 